\title{
J oint Investigation of Two-Month Post-Diagnosis IgG Antibody Levels and Psychological Measures for Assessing Longer Term Multi-Faceted Recovery among COVID-19 Cases in Northern Cyprus
}

Burc Barin ${ }^{1 *}$, Banu E. Yoldascan ${ }^{2}$, Fatma Savaskan ${ }^{3}$, Goncagul Ozbalikci ${ }^{4}$, Tugce Karaderi ${ }^{5}$, Huseyin Çakal ${ }^{6}$

${ }^{1}$ The Emmes Company, United States, ${ }^{2}$ Department of Public Health, Faculty of Medicine, Cyprus International University, Cyprus, ${ }^{3}$ Infection Control Unit, Burhan Nalbantoglu State Hospital, Cyprus, ${ }^{4}$ Microbiology Laboratory, Burhan Nalbantoglu State Hospital, Cyprus, ${ }^{5}$ Disease Systems Biology Program, Novo Nordisk Foundation Center for Protein Research, Faculty of Medical and Health Sciences, University of Copenhagen, Denmark, ${ }^{6}$ School of Psychology, Keele University, United Kingdom

Submitted to J ournal:

Frontiers in Public Health

Specialty Section:

Infectious Diseases - Surveillance, Prevention and Treatment

Article type:

Brief Research Report Article

Manuscript ID:

590096

Received on:

31 J ul 2020

Revised on:

19 Oct 2020

Frontiers website link:

www. frontiersin.org 


\section{Conflict of interest statement}

\section{The authors declare that the research was conducted in the absence of any commercial or financial relationships that could be construed as a potential conflict of interest}

\section{Author contribution statement}

Elcin Yoldascan, Burc Barin, Fatma Savaskan planned the study and its implementation.

Burc Barin, Huseyin Cakal, Elcin Yoldascan and Fatma Savaskan designed the psychological survey.

Fatma Savaskan coordinated the fieldwork for collection of blood samples, administration of psychological surveys and compilation of survey data.

Goncagul Ozbalikci coordinated processing of blood samples, running of immunoassays and compilation of the assay data.

Burc Barin and Huseyin Cakal performed the statistical analyses.

Burc Barin wrote the first draft.

Tugce Karaderi and Huseyin Cakal conducted critical review/ editing for the major revision.

Burc Barin, Tugce Karaderi, Huseyin Cakal conceptualized, revised and finalized the article.

All authors have reviewed the article, provided feedback and approved the article for publication.

\section{Keywords}

COVID-19, SARS-CoV-2, Recovery, immune response, antibody, Psychological impact, Trauma, stigma

\section{Abstract}

Word count:

250

Following the outbreak of COVID-19, multidisciplinary research focusing on the long-term effects of the COVID-19 infection and the complete recovery is still scarce. With regards to long-term consequences, biomarkers of physiological effects as well as the psychological experiences are of significant importance for comprehensively understanding the complete COVID-19 recovery period. The present research surveys the IgG antibody titers and the impact of COVID-19 as a traumatic experience in the aftermath of the active infection period, around two months after diagnosis, in a subset of COVID-19 patients from the first wave (March-April 2020) of the outbreak in Northern Cyprus. Associations of antibody titers and psychological survey measures with baseline characteristics and disease severity were explored, and correlations among various measures were evaluated. Of the 47 serology tests conducted for presence of IgG antibodies, 39 (83\%) were positive. We identified trends demonstrating individuals experiencing severe or critical COVID-19 disease and/ or those with comorbidities are more heavily impacted both physiologically and mentally, with higher IgG titers and negative psychological experience compared to those with milder disease and without comorbidities. We also observed that more than half of the COVID-19 cases had negative psychological experiences, being subjected to discrimination and verbal harassment/ insult, by family/friends. In summary, as the first study co-evaluating immune response together with mental status in COVID-19, our findings suggest that further multidisciplinary research in larger sample populations as well as community intervention plans are needed to holistically address the physiological and psychological effects of COVID-19 among the cases in the long-term.

\section{Contribution to the field}

Our study is important as it is the first study jointly evaluating post-discharge blood antibody levels and psychological status of COVID-19 cases at a median time of two months after diagnosis. Severe/ critical COVID-19 cases had higher blood IgG antibody levels as well as the highest long-term mental impact. Holistic and a more personalized approach is needed for post-discharge monitoring and treatment of COVID-19 cases, with a focus on older age, comorbidity status and disease severity. 


\section{Ethics statements}

\section{Studies involving animal subjects}

Generated Statement: No animal studies are presented in this manuscript.

\section{Studies involving human subjects}

Generated Statement: The studies involving human participants were reviewed and approved by The study has been reviewed and approved by the International Cyprus University Ethics Committee in Nicosia, Cyprus. . Written informed consent to participate in this study was provided by the participants' legal guardian/ next of kin.

\section{Inclusion of identifiable human data}

Generated Statement: No potentially identifiable human images or data is presented in this study. 


\section{Data availability statement}

Generated Statement: The raw data supporting the conclusions of this article will be made available by the authors, without undue reservation. 


\section{| Joint Investigation of Two-Month Post-Diagnosis, IgG Antibody Levels and Psychological Measures for Assessing Longer Term Multi-Faceted Recovery among COVID-19 Cases in Northern Cyprus}

\author{
Authors: \\ Burc Barin*, Banu Elcin Yoldascan, Fatma Savaskan, Goncagul Ozbalikci, Tugce \\ Karaderi $^{* *}$, Hüseyin Çakal ${ }^{* *}$. \\ * corresponding author \\ ** co-senior authors
}

\section{Abstract:}

Following the outbreak of COVID-19, multidisciplinary research focusing on the longterm effects of the COVID-19 infection and the complete recovery is still scarce. With regards to long-term consequences, biomarkers of physiological effects as well as the psychological experiences are of significant importance for comprehensively understanding the complete COVID-19 recovery, The present research surveys the IgG antibody titers and the impact of COVID-19 as a traumatic experience in the aftermath of the active infection period, around two months after diagnosis, in a subset of COVID-19 patients from the first wave (March-April 2020) of the outbreak in Northern Cyprus. Associations of antibody titers and psychological survey measures with baseline characteristics and disease severity were explored, and correlations among various measures were evaluated. Of the 47 serology tests conducted for presence of IgG antibodies, $39(83 \%)$ were positive. We identified trends demonstrating individuals experiencing severe or critical COVID-19 disease and/or those with comorbidities are more heavily impacted both physiologically and mentally, with higher IgG titers and negative psychological experience compared to those with milder disease and without comorbidities. We also observed that more than half of the COVID-19 cases had negative psychological experiences, being subjected to discrimination and verbal harassment/insult, by family/friends. In summary, as the first study co-evaluating immune response together with mental status in COVID-19, our findings suggest that further multidisciplinary research in larger sample populations as well as community intervention plans are needed to holistically address the physiological and psychological effects of COVID-19 among the cases,

Keywords: COVID-19, SARS-CoV-2, recovery, immune response, antibody, psychological impact, trauma, stigma.

\section{Section I. Introduction}

Coronavirus disease of 2019 (COVID-19), resulting from SARS-CoV-2 infection, was declared a pandemic by the World Health Organization on 11 March 2020. As of 29 July 2020, more than 16,000,000 COVID-19 cases were identified, and more than 650,000 deaths were reported due to the disease (1). Although the scientific community has responded rapidly to detect the transmission mechanisms and develop vaccines, multidisciplinary research focusing on the long-term effects of the COVID-19 infection is still scarce, and not much is known on how the human body responds to COVID-19

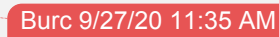

Comment [1]: Corrected error from first version.

Burc 8/27/20 1:14 PM

Deleted: Discharge

\begin{tabular}{l}
\hline Burc 9/27/20 11:34 AM \\
Comment [2]: Revised per Comment by \\
Reviewer \#1. \\
Burc 9/27/20 10:59 PM \\
\hline Deleted: ' \\
\hline Burc 9/27/20 11:34 AM \\
\hline Deleted: longer term \\
\hline Burc 9/27/20 10:59 PM \\
\hline Deleted: \\
\hline Burc 9/27/20 10:59 PM \\
\hline Deleted: whole \\
\hline Burc 9/27/20 11:00 PM \\
\hline Deleted: period \\
\hline Burc 9/27/20 11:36 AM \\
\hline Comment [3]: Corrected error from the \\
first version. \\
\hline Burc 8/27/20 1:14 PM \\
\hline Deleted: discharge \\
\hline Burc 9/27/20 12:00 PM \\
\hline Comment [4]: Added in per Comment by \\
Reviewer \#1. \\
\hline Burc 9/27/20 11:44 AM \\
\hline Comment [5]: Clarified per Comment by \\
Reviewer \#1. \\
\hline Burc 9/27/20 11:44 AM \\
\hline Comment [6]: Removed “long-term" per \\
the Comment by Reviewer \#1. \\
\hline Burc 9/27/20 11:43 AM \\
\hline Deleted: in the long-term \\
\hline
\end{tabular}


infection, both biologically and psychologically during the 'longer term recovery' period after discharge from the hospital/isolation. With regards to long-term effects, biomarkers of physiological effects as well as the psychological experiences are of significant importance for a comprehensive understanding of the COVID-19 recovery period (2). COVID-19 as a life-threatening infection can act as an acute stressor (3) and stress can have a down-regulatory effect on the immune system (4). The present research surveys the IgG antibody titers and the impact of COVID-19 as a traumatic experience both during and in the aftermath of the active infection period.

There is insufficient information on the immune response to COVID-19 (e.g. prevalence of different antibodies against the infection over time and development of long-term immunity). It is essential to better understand the timeline of immune response including the appearance of immunoglobulin $\mathrm{M}(\operatorname{IgM})$ and immunoglobulin $\mathrm{G}(\operatorname{IgG})$ antibodies, their lifespan and whether they are protective, at least partially, against a second infection. Preliminary research shows that detectable $\operatorname{IgG}$ antibodies generally start appearing after the first week after symptom onset, reach a peak around two to three weeks, and stay at detectable blood levels at least for a duration of 2-3 months even in milder cases, similar to previous observations in other SARS infections (5-7). Moreover, the psychological effects of having the infection are also complex. The potential lifethreatening impact of having severe COVID-19, the overall disease burden, along with many unknowns about its short- and long-term effects jncrease the stigma attached to the infection and the related anxiety among the public., These factors, in turn make COVID19 cases more vulnerable to post-traumatic stress as well as targets for harassment and discrimination (8). It is presumed that the period of complete physiological and psychological recovery from the infection depends on disease severity and other physiological and socioeconomic factors. However, given all the elaborate aspects of COVID-19 yet to be investigated and understood, the multi-faceted complete recovery period is still far from being deciphered.

From a psychological point of view, initial findings suggest that both the disease itself and the negative consequences of the lockdown imposed by governments to curb the spread of the disease could result in negative coping behaviour which includes but is not limited to panic, anxiety, stigmatization, and post-traumatic stress disorder (PTSD) (3). As scarce research shows, these reactions can also be influenced by contextual factors such as a history of war, famine, natural disasters, man-made accidents and the size of the population. More specifically, while smaller nations might appear to have the upper hand in rapid enforcement of measures, contextual factors such as the increased connectivity of the individuals in smaller societies, or negative collective experiences of war and famine in the past might increase the prevalence of negative coping behaviours and stigma induced depression (9).

A particular case in point is Northern Cyprus, governed by a state that remains internationally unrecognized, and hence, not included in the global epidemiological COVID-19 statistics. In the first wave of the COVID-19 outbreak in Northern Cyprus, 108 cases were diagnosed between 10 March and 16 April 2020. The authorities responded promptly and lockdown was imposed on March 11 (9) effectively halting
Burc 9/27/20 12:02 PM

Comment [7]: To address question by Reviewer \#1: Correct, 'discharge' in our analysis refers to either discharge from hospital or isolation - this has been further defined/clarified in the methods section. Burc 10/17/20 3:44 PM

Deleted:

\begin{tabular}{l}
\hline Burc 9/27/20 12:12 PM \\
\hline Deleted: risk \\
\hline Burc 9/27/20 12:08 PM \\
\hline Deleted: \\
\hline Burc 9/27/20 12:13 PM \\
\hline Deleted: and \\
\hline Burc 9/27/20 12:14 PM \\
\hline $\begin{array}{l}\text { Comment [8]: Clarified and split the } \\
\text { sentence into two parts per Comment by } \\
\text { Reviewer \#1. }\end{array}$ \\
\hline Burc 9/27/20 12:05 PM \\
\hline $\begin{array}{l}\text { Comment [9]: Revised per comment by } \\
\text { Reviewer \#1. }\end{array}$ \\
\hline Burc 9/27/20 12:05 PM \\
\hline Deleted: long-term \\
\hline Burc 9/27/20 12:16 PM \\
\hline $\begin{array}{l}\text { Comment [10]: Added in per Comment } \\
\text { by Reviewer \#1. }\end{array}$ \\
\hline
\end{tabular}


education and government offices, and all other services except those considered essential. In addition to the global concern over the pandemic, the small community setting in Northern Cyprus (an estimated total population of around 400,000) with a history of war and trauma (10) further caused intensified anxiety and fear in an already sensitive population, Panic engulfed the small nation and there was widespread stigma toward those who tested positive or considered high-risk for transmitting the disease, i.e. Turkish Cypriots living abroad, who were brought home and quarantined (11). Videos of individuals under duress as a result of being quarantined were widely circulated in the social media ${ }_{2}$ and there were news of occasional small-scale protests in neighbourhoods where quarantine hotels were chosen due to the perceived infection threat (12). Similarly, those who were tested positive recounted psychological trauma as their names made public and have been targeted (13). Therefore, there is sufficient grounds to assume that in addition to the physiological impact of the disease, those who tested positive for the COVID-19 have also experienced psychological distress during and after the active infection period. In fact, in an earlier study conducted in Wuhan (China), the prevalence of significant post-traumatic stress symptoms associated with COVID-19 was estimated as $96.2 \%$ among clinically stable COVID-19 cases at discharge from quarantine (14). Taken together, these observations suggest that assessing the biological markers of physiological effects vis-à-vis negative psychological experiences of the COVID-19 cases is important for holistic management of COVID-19 patients from diagnosis to potentially complete physiological and psychological recovery. The present research surveys the immune response (IgG antibody titers) and negative psychological experiences among the COVID-19 cases in the complete, recovery period in the small society setting of Northern Cyprus.

\section{Section II. Sample Population and Methodology}

\section{Participants and Study Design}

We performed a joint investigation of the immune response and mental status of the COVID-19 cases at an average time of two months after diagnosis, Within the scope of our study, these two main outcomes of interest comprise the assessments toward the complete, recovery of the cases. Of the 108 cases diagnosed, 32 were tourists on the island: two died with the disease, and the remaining 30 individuals returned to their country after discharge from hospital/isolation. Dependent on the severity of the disease, COVID-19 cases were either monitored in the hospital or isolation hotels designated by the health authority. Of the remaining 76 individuals residing in Northern Cyprus, two died with the disease. A total of 74 individuals were invited to participate in the postdischarge assessment of antibody development and psychological impact. For the psychological evaluation, eight individuals under the age of 18 as well as three individuals who did not speak Turkish or English fluently were excluded from the study. Hence, a total of sixty-three individuals were eligible to participate in the psychological evaluation.

\begin{tabular}{|c|}
\hline Deleted: scare about \\
\hline Burc 10/2/20 11:22 PM \\
\hline Deleted: an \\
\hline Burc 10/2/20 11:21 PM \\
\hline Deleted: disease stigm \\
\hline Burc 10/2/20 11:21 PM \\
\hline Deleted: a. On the one hand, $\mathrm{p}$ \\
\hline Burc 10/2/20 11:29 PM \\
\hline Deleted: On the other hand, v \\
\hline Burc 10/2/20 11:36 PM \\
\hline $\begin{array}{l}\text { Comment [11]: Revised these sentences } \\
\text { to flow better per comments by Reviewer } \\
\# 1 \text {. }\end{array}$ \\
\hline Burc 10/2/20 11:30 PM \\
\hline Deleted: the public reacted negatively \\
\hline Burc 10/17/20 3:45 PM \\
\hline Deleted: \\
\hline Burc 9/28/20 2:10 AM \\
\hline Deleted: \\
\hline Burc 10/17/20 3:59 PM \\
\hline $\begin{array}{l}\text { Comment [12]: Revised to a consistent } \\
\text { terminology per comment by Reviewer \#1. }\end{array}$ \\
\hline Burc 10/17/20 3:57 PM \\
\hline Deleted: extended \\
\hline Burc 9/27/20 11:47 AM \\
\hline $\begin{array}{l}\text { Comment [13]: Corrected the error in the } \\
\text { first version. }\end{array}$ \\
\hline Burc 8/27/20 1:15 PM \\
\hline $\begin{array}{l}\text { Deleted: discharge from the } \\
\text { hospital/isolation }\end{array}$ \\
\hline Burc 10/17/20 3:46 PM \\
\hline Deleted: $\mathrm{T}$ \\
\hline Burc 10/17/20 3:59 PM \\
\hline Deleted: longer term \\
\hline Burc 9/27/20 11:56 AM \\
\hline $\begin{array}{l}\text { Comment [14]: Per Comment by } \\
\text { Reviewer \#1, we further defined in this } \\
\text { sentence what 'discharge' refers to in the } \\
\text { regional context. }\end{array}$ \\
\hline Burc 9/27/20 12:39 PM \\
\hline $\begin{array}{l}\text { Comment [15]: Clarified per Comment by } \\
\text { Reviewer \#1. }\end{array}$ \\
\hline Burc 9/27/20 12:39 PM \\
\hline Deleted: / \\
\hline
\end{tabular}


All subjects were informed about both components of the study, provided informed consent acknowledging voluntary participation, option to withdraw from study at any time, and the confidentiality of their antibody results and their responses to the survey.

\section{Eligibility Criteria}

General Inclusion Criteria: Confirmed (i.e., with positive polymerase chain reaction test result) COVID-19 infection in Northern Cyprus between the dates of 10 March - 17 April and residence in northern Cyprus.

Exclusion Criteria for Antibody Development Analysis: Refusal to give informed consent, or contraindication to venipuncture.

Exclusion Criteria for Psychological Survey: Refusal to give informed consent, inability to understand/speak Turkish or English fluently, or being under the age of 18 .

\section{Blood Collection and Transfer}

Blood samples were taken by trained nurses during home visits. Venipuncture was used to collect blood. $10 \mathrm{ml}$ complete gel barrier formation tubes were used for blood collection (See Supplementary Text for the details).

\section{Serology Testing}

The Abbott SARS-CoV-2 IgG assay is a chemiluminescent microparticle immunoassay (CMIA) intended for both the quantitative and qualitative detection of IgG antibodies to the nucleocapsid protein of SARS-CoV-2 in human blood serum and plasma. Assay specifications indicate that the SARS-CoV-2 IgG assay is intended for use as an aid in identifying individuals with an adaptive immune response to SARS-CoV-2, indicating recent or prior infection. This assay is only for use under the United States Food and Drug Administration's Emergency Use Authorization. Per the assay's recommended definition, we defined positive IgG response in the study as a titer level $\geq 1.4$ index signal/cutoff (s/co) (15). Assays were run on Abbott's ARCHITECTPlus i2000 ${ }_{\mathrm{SR}}$ System.

The reported positive predictive agreement (PPA) for the assay at $\geq 14$ days postsymptom onset was $100.0 \%$ (95\% confidence-interval (CI): $95.9 \%-100 \%)$ while the negative predictive agreement (NPA) was 99.6\% (95\% CI: 99.1\%-99.9\%). Performance characteristics of the assay were independently evaluated in a study conducted in Boise, Idaho, where specificity and sensitivity were reported as $99.90 \%$ and $100 \%$ (starting at day 17 after symptom onset), respectively (16).

\section{Psychological Measures}

We designed a questionnaire-based survey to assess the negative psychological experiences of the cases. Whenever possible, we adapted and used tested and validated measures for known psychological processes, More specifically, we assessed the extent of experiencing COVID-19 as a life changing trauma (CALCT), negative emotions, perceived importance of preventive measures, awareness and habits, initial reaction to diagnosis, evaluation of general health, stigma, perceived discrimination, post-traumatic anxiety, and evolving perspectives after discharge via the survey response measures. Ordinal response scales with five levels (with corresponding scores of 1-5) were used for each measure, Multiple measures on the same psychological process were combined to

\section{Burc 9/27/20 12:41 PM}

Comment [16]: Reviewer \#1 inquired about location of Supplementary Text. This was uploaded separately at the time of submission, along with Supplementary Tables/Figures. Burc 9/27/20 12:42 PM

Comment [17]: Added in per Comment by Reviewer \#1.

\section{Burc 10/3/20 1:36 AM}

Deleted: already Burc 10/3/20 12:20 AM

Deleted: to assess the negative psychological experiences of the cases Burc 9/27/20 12:52 PM

Comment [19]: Moved up the introduction of the abbreviation per Comment by Reviewer \#1. Burc 10/3/20 1:53 AM 
create one composite scale process measure by computing the average score per individual. Higher computed scores, indicated stronger experience of COVID-19 as a life changing trauma, perceived higher importance of preventive measures, stronger initial reaction to diagnosis, more positive evaluation of general health, more perceived discrimination, higher post-traumatic anxiety, and stronger anticipation of future COVID19 related anxiety.

We verified the internal reliability of our multi-item process measures via Chronbach's alpha $(\alpha>0.70)$. Experiencing COVID-19 as a life-changing traumatic was measured with three items $(\alpha=0.84)$ adapted from (17). Negative emotions during the recovery were assessed by four items $(\alpha=0.79)$. Perceived discrimination on the basis of being COVID19 positive was measured by six items $(\alpha=0.90)$ adapted from (18). We also measured anxiety related to anticipated stigma in the future as a result of COVID-19 diagnosis with two items $(\mathrm{r}=0.82, \mathrm{p}<0.001)$. We measured subjective evaluation of health before the diagnosis and after the discharge with a single item each. Willingness to help others by sharing information was measured by a single item and perceived importance of protective measures by 4 items $(\alpha=0.96)$. Full list of the items can be found in the Supplementary Material - Psychological Survey.

\section{Statistical Analysis}

Analysis of quantitative IgG titers and CALCT psychological process scores was conducted via non-parametric tests: Wilcoxon rank-sum test (for factors with two levels) and Kruskal-Wallis test (for factors with three or more levels). Due to small group sample sizes, these rank-based non-parametric tests that do not make any assumptions regarding the underlying distribution of the data were preferred for group comparisons (19-20). We computed descriptive statistics for the socio-demographics factors and summary measures (mean score (M) with standard deviation (SD)) for psychological processes, and conducted Pearson correlation tests to explore whether the selected psychological processes were associated with each other. All single-item survey questions and multi-item process measures use 5-point Likert scales (1 lowest, 5 highest) and have a mid-level at 2.5. Disease severity was defined as critical (requiring intensive care), severe (requiring oxygen therapy, but otherwise stable) and mild/moderate (all other cases). P-values less than 0.001 were displayed as " $p<0.001$ ". Statistical significance was defined as $\mathrm{p}<0.05$. Multivariate analyses were not carried out due to small sample size. Analyses were performed using SAS version 9.4 (SAS, Cary, NC, USA).

\section{Section III. Results}

\section{Baseline Characteristics}

Of the 74 cases eligible for serology testing, $47(64 \% ; 60 \%$ women and $40 \%$ men) accepted the invite and provided blood for testing. Median [interquartile range (IQR)] time from initial COVID-19 diagnosis to blood draw for serology testing was 66 [63.573] days with min-max of 50-86 days. Of the 63 cases eligible for responding to the psychological survey, 41 (65\%) responded to survey questions (Table 1).

For the serology testing, $19 \%$ were $<30$ years of age, $53 \%$ were between $30-60$ years old,
Burc 10/3/20 2:19 AM

Deleted: values

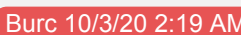

Deleted: assessed

\section{Burc 10/3/20 1:58 AM}

Deleted: ; See Supplementary Material -

Psychological Survey for the full

Questionnaire

\section{Burc 10/20/20 1:21 AM}

Comment [20]: We have re-written the Psychological Processes section to more clearly define the computation of the derived process scores and to make the flow better/clearer in order to address Comment by Reviewer \#2.

\begin{tabular}{l} 
Burc 10/19/20 10:38 PM \\
Comment [21]: To address a comment by \\
Reviewer \#2, we added further clarification \\
and references on the statistical tests used \\
for group comparisons. \\
Burc 10/19/20 10:36 PM \\
Formatted: Font color: Red \\
Burc 10/19/20 10:36 PM \\
Formatted: Font color: Red \\
Burc 10/4/20 8:38 AM \\
Deleted: and \\
Burc 10/4/20 8:37 AM \\
Deleted: single items \\
Burc 10/4/20 8:46 AM \\
Formatted: English (US) \\
\hline Burc 9/27/20 12:48 PM \\
Comment [22]: Corrected an error from \\
the first version. \\
\hline Burc 8/27/20 1:18 PM \\
Deleted: 4 \\
\hline
\end{tabular}


and $28 \%$ were $\geq 60$ years of age. At the time of COVID-19 diagnosis, $79 \%$ and $47 \%$ of the serology analysis participants reported 'at least one symptom' and 'fever history', respectively. Thirty-two percent had at least one comorbidity - most frequently hypertension $(\mathrm{N}=9)$ and diabetes $(\mathrm{N}=5$, two with concurrent hypertension). COVID-19 disease severity was severe or critical for $9(19 \%)$ cases and mild/moderate for the remaining $38(81 \%)$. For the psychological survey, distributions of participant baseline and disease severity characteristics were similar to those of the blood serology analysis (Table 1). Detailed cross-tabulation of baseline characteristics and disease severity by age group is displayed in Supplementary Table 1.

\section{Serology}

Of the 47 serology tests conducted for IgG antibody development, 39 (83\%) were positive and $8(17 \%)$ were negative. All of the negative results came from individuals who experienced mild/moderate disease. Overall median [IQR] titer level was 4.38 [2.055.88]. Median [IQR] titer level among positives and negatives were 4.95 [3.79-6.09] and 0.61 [0.16-0.72], respectively.

Figure 1 and Supplementary Table 2 display the distribution of IgG antibody titers by baseline characteristics and disease severity. The factor that had the most impact on IgG titer at a median follow-up of two months post-diagnosis was disease severity. Nine subjects who had severe/critical disease had median [IQR] IgG titer of 6.09 [5.88-6.24] versus 3.94 [1.70-5.52] reported for thirty-eight subjects with mild/moderate disease (Wilcoxon rank-sum test; $\mathrm{p}=0.001$ ). Among the baseline factors, fever/history of fever reported at the time of diagnosis yielded median [IQR] IgG titer of 5.56 [4.11-6.20] versus 3.57 [1.47-5.13] reported for those without fever/history of fever (Wilcoxon ranksum test; $\mathrm{p}=0.01$ ). Having a comorbidity also produced higher median [IQR] IgG titers of 5.52 [4.31-6.09] versus 3.87 [1.25-5.56] in those without a comorbidity (Wilcoxon rank-sum test; $\mathrm{p}=0.03$ ).

The distributions of IgG titers by cross-tabulation of baseline characteristics and disease severity are displayed in Supplementary Table 3. In the mild/moderate disease severity group, a significantly higher level of $\operatorname{IgG}$ titer was observed in individuals with comorbidities (median [IQR]: 5.02 [3.92-5.67]) compared to those without (median [IQR]: 3.43 [0.88-4.87]) (Wilcoxon rank-sum test; $\mathrm{p}=0.03$ ).

\section{Negative Psychological Experiences}

We report the descriptive statistics and the associations between negative psychological processes in Table 2.

Perception of COVID-19 diagnosis as a life changing traumatic event revealed a mean score of 3.17 [SD 1.41], which is above the mid-level. Figure 2 displays the distribution of CALCT scores by baseline characteristics and disease severity. Similar to the IgG titer analysis, the factors that have shown trends for the most impact on CALCT scores at a median follow-up of two months post-diagnosis was disease severity, followed by presence of a comorbidity. Mean (SD) CALCT scores in mild/moderate and severe/critical disease groups were 3.01 (1.38) and 3.95 (1.42), respectively (Wilcoxon rank-sum test; $\mathrm{p}=0.10$ ). For individuals with a comorbidity, mean (SD) CALCT score 
was 3.53 (1.48) as compared to 3.02 (1.39) in those without (Wilcoxon rank-sum test; $\mathrm{p}=0.30)$.

Among the individual questions measuring the negative psychological experiences, twenty-four (59\%) respondents indicated a change in their perspective on life and their priorities due to the COVID-19 infection. All six (100\%) responders to the question with severe/critical disease and 18 out of $33(55 \%)$ responders with mild/moderate disease indicated a change in their perspective on life and their priorities due to the COVID-19 infection. $19(46 \%)$ individuals indicated that they have become a more worried/anxious person because of the infection, and $20(49 \%)$ perceived the infection period as a turning point in their lives $(42 \%$ and $75 \%$ of the individuals with mild/moderate and severe/critical disease, respectively) (Supplementary Table 4).

The mean score for the negative emotions due to COVID-19 diagnosis was 2.61 (SD 1.25 ) and also above the mid-level of the scale (2.5). As for the individual emotions, felt as an initial reaction to COVID-19 diagnosis, worry ranked the first with $71 \%$ of respondents having felt it moderately, a lot or quite a lot, followed by helplessness $(47 \%)$, fear of death (31\%) and guilt due to not being sufficiently self-protected (19\%). Fear of death and helplessness were both reported moderately or above by $27 \%$ and $50 \%$ of individuals in the mild/moderate and severe/critical disease severity groups, respectively (Supplementary Table 5).

Additional analyses of our psychological measures revealed that perceiving COVID-19 as a life changing trauma is strongly and positively associated with experiencing negative emotions $(\mathrm{r}=0.54, \mathrm{p}<0.001)$; perceived discrimination $(\mathrm{r}=0.54, \mathrm{p}<0.001)$; and future stigma related anxiety $(\mathrm{r}=0.54, \mathrm{p}<0.001)$. Similarly, perceived importance of protective measures is again strongly and positively associated with pro-social tendencies $(\mathrm{r}=0.41$, $\mathrm{p}<0.001)$. Last but not least, perceived discrimination at present is strongly and positively associated future stigma related anxiety $(\mathrm{r}=0.80, \mathrm{p}<0.001)$ (Table 2).

\section{Section IV. Conclusions}

We detected IgG antibodies in 39 (out of 47;83\%) of cases after a median of 66 days, which was a considerably longer follow-up period compared to the previous serological studies on IgG (on average up to $\sim 30$ days; 21-23). This observation confirms that IgG antibodies are still detectable in the blood in most COVID-19 cases around 2 months post-diagnosis. However, further studies are necessary to determine the neutralizing activity of these antibodies and whether they provide any immunity against a second infection. Moreover, severe/critical COVID-19 cases most of whom were older and/or with comorbidities had higher IgG titers, and also showed trends for the most impact mentally. Overall, we conclude that more specialized attention should be paid to this group for providing further monitoring and treatment post-discharge because of their higher healthcare needs related to comorbidities as well as the psychological impact in order to expedite the full recovery period after the infection. 
Our analyses replicated the previous observations that disease severity is an important predictor of blood IgG levels (21-23), and confirmed that this observation holds true in the longer follow-up period we examined. Furthermore, among individuals with mild or moderate disease, we observed that those with comorbidities had significantly higher IgG levels (Supplementary Table 3). Similarly, Liu et al. observed that besides the severe COVID-19 cases who tended to have a more vigorous IgG response, a subset of the cases with mild disease had a robust $\operatorname{IgG}$ antibody response, and suggested that age and comorbidities may impact the timing and magnitude of the immune response (23). Fever reported at the time of diagnosis also hinted at a possible association with postdischarge IgG levels, but studies with larger case numbers are needed to evaluate these potential predictors of IgG levels with respect to potential confounders such as age, sex, different types of co-morbidities (e.g. autoimmune and endocrine-related diseases) and disease severity via multivariate models. All these factors with potential association to higher IgG titers are correlated with each other, and reflect increased disease burden during diagnosis and post-discharge (Supplementary Table 1). It is known that severity of COVID-19 is associated with a dysregulated immune response, and hence, further investigation of how dysregulated immune response is reflected in the long-term blood antibody levels may provide insights into the biological mechanism of the disease and support development of effective vaccines that are based on long-term immune response $(24,25)$.

In line with previous research, one in every two individuals with severe/critical disease felt fear of death and helplessness while one in every four individuals with mild/moderate disease felt these two emotions. Worry was the most commonly expressed emotion among the four negative emotions queried, with $71 \%$ of respondents having felt it moderately or more (Supplementary Table 5). Based on the responses to the psychological survey about two months after diagnosis, we infer that most cases have not yet recovered from the mental impact of the disease. Participants experienced COVID-19 as a life-changing trauma, experienced negative emotions, perceived themselves as discriminated against and experienced anxiety due to anticipated stigma in the future. In addition to replicating previous research on the negative psychological consequences of being tested positive for an infectious disease and that pandemics have a lasting negative impact on mental health among the general population (26-28), our findings also show that cases experienced anxiety as a result of anticipated stigma. This is a novel finding which reveals that pandemics like COVID-19 have long-term negative mental health effects. Future research could replicate and extend these findings via longitudinal designs.

Post-traumatic stress is an important part of this disease due to its overall severity, global impact and stigma attached to it. About half of the survey respondents reported being a more worried person due to the infection, and perceiving the infection as a turning point in their life. About one in four individuals also reported concern that their relationship with their workplace and family/friends will deteriorate due to infection. Hence, community resources for provision of psychological support to the COVID-19 cases post-discharge is very important to minimize the long-term impact of the disease and maintain mental health in these individuals. In Northern Cyprus, a number of
Burc 10/19/20 10:39 PM

Deleted: 19

Burc 10/19/20 10:39 PM

Deleted: 21

Burc 10/19/20 10:40 PM

Deleted: 21

\section{Burc 10/19/20 10:40 PM}

Deleted: 22

Burc 10/19/20 10:40 PM

Deleted: 23

Burc 10/4/20 10:04 AM

Deleted: five

Burc 10/4/20 10:18 AM

Comment [23]: Clarified reference to

worry per a comment by Reviewer \#1.

\section{Burc 10/19/20 10:40 PM}

Deleted: 24

Burc 10/19/20 10:40 PM

Deleted: 26 
organizations and universities have already taken action and set up psychological counseling hotlines, free for use by the public (9). These initiatives are very important and need to be expanded throughout the counties, regions and globally. However, more tailored intervention programs are needed especially for COVID-19 cases to combine mental check-ups with regular health check-ups at regular intervals. About one in ten individuals thought they could still transmit the disease. This provides another example of importance of using up-to-date medical info about the disease, and the person's current status in providing tailored therapy to the person for getting over pre-conceived notions about fear of continuing disease in the individual.

Overall perception about the disease as a threat varied with disease severity (Supplementary Table 12). While more than half of the cases with mild/moderate disease deemed the infection was nothing to be afraid of, only one in four thought the same among the severe/critical cases. Therefore, a consistent public communication strategy is needed to ensure public perception of the disease will not change over time from a conscious alertness to the disease being 'nothing to be afraid of' due to sharing of experiences/perceptions by an estimated $80 \%$ of the cases in the mild/moderate severity group among the community.

435

The study is subject to a number of limitations. Although our study participation rates of 64\% (serology) and 65\% (psychology) among discharged COVID-19 cases are acceptable for an exploratory study such as this one there may be some differences between individuals willing and unwilling to participate in the study, especially with respect to psychological endpoints. Actually, we observed lower participation rates in the study by cases from a rural region that was more severely impacted by the outbreak and had to go under a regional quarantine for an extended time. Disease stigma, continuing worry, suspicion and mistrust likely led to lower participation rates, and these factors are directly related to psychological endpoints studied here. To facilitate a more practical implementation in the field, it was not possible to use a consistent time point for evaluation of the outcomes of interest. Nevertheless, timing of blood draw and survey response showed limited variability around a two-month time point post-diagnosis, with median [IQR] and range time being 66 [63.5-73] and 50-86 days, respectively. Due to limited resources, it was not possible to conduct the study longitudinally via multiple time points to evaluate trends in further detail. There were possibly correlated responses for either or both endpoints as we allowed participation of multiple family or household members in the study. There were eight families that were represented in the study with 2-3 members each. Finally, compared to continuous $\operatorname{IgG}$ titer measures, categorical nature of the survey responses produced higher variability in calculated psychological process scores, and hence, lower statistical power in detecting any associations with baseline factors and disease severity. symptoms or measures that are potentially associated with continuing recovery process. At the time we conducted our study, little was known on the long-term impact of 461 COVID-19 and how it manifested in the cases. In recent months, there has been evolving 462 information regarding numerous defined and undefined conditions associated with

\begin{tabular}{l} 
Burc 9/27/20 11:49 AM \\
Comment [24]: Corrected an error in the \\
first version. \\
Burc 8/27/20 1:31 PM \\
Deleted: 4 \\
\hline Burc 9/27/20 11:20 PM \\
Deleted: \\
\hline Burc 9/27/20 11:20 PM \\
Deleted: $T$ \\
\hline
\end{tabular}

Burc 10/4/20 9:55 AM

Comment [25]: Added in this limitation per a Comment by Reviewer \#2. 
COVID-19, including long-term organ damage, nervous system damage and immune system dysregulations. The most commonly reported general symptoms post acute COVID-19 have been fatigue and dyspnea, followed by joint pain and chest pain. As part of the organ-specific dysfunction, myocardial injury/inflammation has been detected via increased troponin levels and cardiac magnetic resonance imaging, and pulmonary dysfunction via radiologic abnormalities, decreased diffusion capacity for carbon monoxide and diminished respiratory muscle strength. The most common neurologic symptoms reported have been headache, vertigo, anosmia and ageusia, with encephalitis, seizures, major mood swings and "brain fog" also having been reported (29). A refined and detailed assessment of complete recovery process in future studies should include monitoring for these conditions occurring mostly post-discharge via their associated symptoms, laboratory test results and/or medical imaging findings.

In conclusion, this is the first study jointly evaluating post-discharge blood antibody levels and psychological status at a median time of two months after diagnosis. Severe/critical COVID-19 cases had higher blood IgG antibody levels as well as the highest long-term mental impact. Holistic and a more personalized approach is needed for post-discharge monitoring and treatment of COVID-19 cases, with a focus on older age, comorbidity status and disease severity. Recognizing the long-term impact of the disease (coined as "long COVID"; 30), collaborating globally to accumulate detailed standardized long-term psychological and physiological data (31) and continuing to redefine and publicize the importance of complete recovery are key in addressing the longterm health consequences of COVID-19 via awareness, monitoring and timely intervention,

\section{Acknowledgements}

The authors would like to thank study participants as well as Savas Erdogan, Dr. Sebnem Benar, Safiye Ilgilier, Zeynep Unalan, Cigdem Adatas Sesen, Sumru Ozkan Ezer, Ayse Dogan for helping with the conduct of fieldwork and compilation of study data from psychological surveys, Sedef Kutlu and Serife Can from the microbiology laboratory running the immunoassays, Dr. Selin Özcem, Dr. Zafer Erdoğmuş, Dr. Nesil Bayraktar, Dr. Emre Vudalı, Dr. Mustafa Akansoy, Dr. Emine Kamiloğlu, Dr. Yağmur Aldağ, Dr.

\section{Burc 10/20/20 12:03 AM}

Comment [26]: To address one of the major comments by Reviewer \#2, we added this paragraph to acknowledge the evolving info in this area and the importance of future studies to take these and new info into account. 
Hatice C. Caglayan, Dr. Fatma Canbay and Dr. Derlen O. Rus - the treating physicians who contributed to the baseline characteristics data on study participants, and finally, Dr.

514 Ali Pilli, Dr. Ali Caygur, Dr. Sonuc Buyuk, Dr. Cigdem Caga and Dr. Figen Gulen Ince 515 from the Health Authority in Northern Cyprus for supporting this study.

T.K. was supported by the Novo Nordisk Foundation Center for Protein Research (grant NNF14CC0001).

\section{Data Availability Statement} author.

\section{Ethics Statement}

The study has been reviewed and approved by the International Cyprus University Ethics Committee. The COVID-19 cases i.e. the participants provided their written informed consent to participate in this study.

\section{Author Contributions}

Elcin Yoldascan, Burc Barin, Fatma Savaskan planned the study and its implementation. Burc Barin, Huseyin Cakal, Elcin Yoldascan and Fatma Savaskan designed the psychological survey.

Fatma Savaskan coordinated the fieldwork for collection of blood samples, administration of psychological surveys and compilation of survey data.

Goncagul Ozbalikci coordinated processing of blood samples, running of immunoassays and compilation of the assay data.

Burc Barin and Huseyin Cakal performed the statistical analyses.

Burc Barin wrote the first draft.

Tugce Karaderi and Huseyin Cakal conducted critical review and editing for the major revisions.

Burc Barin, Tugce Karaderi, Huseyin Cakal conceptualized, revised and finalized the article.

All authors have reviewed the article, provided feedback and approved the article for publication.

\section{Conflict of Interest}

The authors declare that the research was conducted in the absence of any commercial or financial relationships that could be construed as a potential conflict of interest.

\section{References}

1. World Health Organization. (2020). Coronavirus disease (COVID19). https://www.who.int/emergencies/diseases/novel-coronavirus-2019/situationreports/ [Accessed July 29, 2020].

2. Holmes, E. A., Connor, R. C. O., Perry, V. H., Tracey, I., Wessely, S., Arseneault, L., ... Bullmore, E. (2020). Multidisciplinary research priorities for the COVID-19 pandemic : a call for action for mental health science. Lancet Psychiatry, 7, 547- 
560. https://doi.org/10.1016/S2215-0366(20)30168-1

3. Dubey, Souvik \& Biswas, Payel \& Ghosh, Ritwik \& Chatterjee, Subhankar \& Dubey, Mahua \& Chatterjee, Subham \& Lahiri, Durjoy \& Lavie, Carl. (2020). Psychosocial impact of COVID-19. Diabetes and Metabolic Syndrome Clinical Research and Reviews. 14. 779-788. 10.1016/j.dsx.2020.05.035.

4. Segerstrom, S. C., \& Miller, G. E. (2006). Psychological Stress and the Human Immune System: A MetaAnalytic Study of 30 Years of Inquiry. Psychological Bulletin, 130(4), 601-630. https://doi.org/doi: 10.1037/0033-2909.130.4.601

5. Sethuraman N., Jeremiah S.S., Ryo A. (2020). Interpreting Diagnostic Tests for SARSCoV-2. JAMA. 2020; 323(22):2249-2251. doi:10.1001/jama.2020.8259

6. Hou, H., Wang, T., Zhang, B., Luo, Y., Mao, L., Wang, F., Wu, S. and Sun, Z. (2020), Detection of IgM and IgG antibodies in patients with coronavirus disease 2019. Clin Transl Immunol, 9: e1136. doi:10.1002/cti2.1136

7. Seow, J., Graham, C., Merrick, B., Acors, S., ..., Doores, K.(2020). Longitudinal evaluation and decline of antibody responses in SARS-CoV-2 infection. medrxiv 2020.07.09.20148429 [Preprint]. Available at: https://doi.org/10.1101/2020.07.09.20148429 (Accessed July 29, 2020).

8. Centers for Disease Control and Prevention. (2020). Coronavirus Disease 2019 (COVID-19).https://www.cdc.gov/coronavirus/2019-ncov/daily-lifecoping/reducing-stigma.html [Accessed July 29, 2020].

9. Volkan, E., \& Volkan, E. (2020). Under the COVID-19 Lockdown : Rapid Review About the Unique Case of North Cyprus. Psychological Trauma: Theory, Research, Practice, and Policy, 12(5), 539-541. https://doi.org/http://dx.doi.org/10.1037/tra0000809

10. Volkan, E., \& Hadjimarkou, M. M. (2019). Undivided Trauma in a Divided Cyprus: Modified Emotional Stroop Study. Psychological Trauma: Theory, Research, Practice, and Policy, Advance on(November 2019). https://doi.org/10.1037/tra0000527

11. Yenidüzen. (2020a). Firat Ataser'e Büyük Tepki. Yenidüzen. Retrieved from http://www.yeniduzen.com/firat-atasere-buyuk-tepki-125200h.htm

12. Yenidüzen. (2020b). Yurt Krizi: Öğrenciler Perişan. Http://Www.Yeniduzen.Com/Yurt-Krizi-Ogrenciler-Perisan-125192h.Htm.

13. Demir, D. (2020). Dışlandık, hain ilan edildik. Yenidüzen. Retrieved from http://www.yeniduzen.com/dislandik-hain-ilan-edildik-129610h.htm

14. Bo, H-X., Li, W., Yang, Y., Wang, Y., Zhang, Q., Cheung, T., Wu, X., Xiang, Y-T. (2020). Posttraumatic stress symptoms and attitude toward crisis mental health services among clinically stable patients with COVID-19 in China. Psychological Medicine 1-2. https:// doi.org/10.1017/S0033291720000999

15. Abbott ARCHITECT SARS-CoV-2 IgG Instructions for Use. H14806R01. April 2020 (https://www.fda.gov/media/137383/download)

16. Bryan, A., Pepper, G., Wener, M.H., et al. (2020). Performance Characteristics of the Abbott Architect SARS-CoV-2 IgG Assay and Seroprevalence in Boise, Idaho. $J$ Clin Microbiol. 58(8):e00941-20. doi:10.1128/JCM.00941-20

17. García, F. E., Cova, F., Rincón, P., Vázquez, C., \& Páez, D. (2016). Coping, rumination and posttraumatic growth in people affected by an earthquake. Psicothema, 28(1), 59-65. https://doi.org/10.7334/psicothema2015.100 
18. Branscombe, N. R., Schmitt, M. T., \& Harvey, R. D. (1999). Perceiving pervasive discrimination among African Americans: Implications for group identification and well-being. Journal of Personality and Social Psychology, 77, 135-149. https://doi.org/10.1037/0022-3514.77.1.135

19. Wilcoxon, F. (1945). Individual comparisons by ranking methods. Biom Bull, 1(6):80-83.

20. Kruskal, W.H., Wallis, W.A. (1952). Use of ranks in one-criterion variance analysis. J. Am. Stat. Assoc. 47, 583-621 and errata, ibid. 48, 907-911.

21, Zhang G., Nie S., Zhang Z., Zhang Z. (2020). Longitudinal Change of Severe Acute Respiratory Syndrome Coronavirus 2 Antibodies in Patients with Coronavirus Disease 2019. The Journal of Infectious Diseases, Volume 222, 2, 15 July 2020, 183-188. https://doi.org/10.1093/infdis/jiaa229

22. Long, Q., Liu, B., Deng, H. et al. (2020). Antibody responses to SARS-CoV-2 in patients with COVID-19. Nat Med, 26, 845-848. https://doi.org/10.1038/s41591020-0897-1

23. Liu, X., Wang, J., Xu, X., Liao, G., Chen, Y. \& Hu, C-H. 2020. Patterns of IgG and IgM antibody response in COVID-19 patients. Emerging Microbes \& Infections, 9:1, 1269-1274. DOI: 10.1080/22221751.2020.1773324

24. Qin, C., Zhou, L., Hu, Z., et al. 2020. Dysregulation of Immune Response in Patients With Coronavirus 2019 (COVID-19) in Wuhan, China, Clinical Infectious Diseases, Volume 71, Issue 15, 1 August 2020, Pages 762-768, https://doi.org/10.1093/cid/ciaa248

25. Diao, B., Wang, C., Tan, Y., Chen, X., Liu, Y., Ning, L., Chen, L., Li, M., Liu, Y., Wang, G., Yuan, Z., Feng, Z., Zhang, Y., Wu, Y., \& Chen, Y. (2020). Reduction and Functional Exhaustion of T Cells in Patients With Coronavirus Disease 2019 (COVID-19). Frontiers in immunology, 11, 827. https://doi.org/10.3389/fimmu.2020.00827

26. Maunder, R., Hunter, J., Vincent, L., Bennett, J., Peladeau, N., Leszcz, M., ... Mazzulli, T. (2003). The immediate psychological and occupational impact of the 2003 SARS outbreak in a teaching hospital. Canadian Medical Association Journal, $168,1245-1251$.

27. Serafini, G., Parmigiani, B., Amerio, A., Aguglia, A., Sher, L., \& Amore, M. (2020). The psychological impact of COVID-19 on the mental health in the general population. QJM: An International Journal of Medicine, 1-7. https://doi.org/10.1093/qjmed/hcaa201

28, Wu, P., Fang, Y., Guan, Z., Fan, B., Kong, J., Yao, Z., ... Hoven, C. W. (2009). The psychological impact of the SARS epidemic on hospital employees in China: Exposure, risk perception, and altruistic acceptance of risk. Canadian Journal of Psychiatry, 54, 302-311. https://doi.org/10.1177/070674370905400504

29. Del Rio, C., Collins, L.F., \& Malani, P. (2020). Long-term Health Consequences of COVID-19. JAMA. doi:10.1001/jama.2020.19719

30. Long COVID: let patients help define long-lasting COVID symptoms. (2020). Nature, 586, 170. https://doi.org/10.1038/d41586-020-02796-2

31. University of Oxford. (2020). Global consortium launches new study into long-term effects of COVID-19. https://www.ox.ac.uk/news/2020-09-11-global-consortiumlaunches-new-study-long-term-effects-covid-19 [Accessed October 18, 2020].

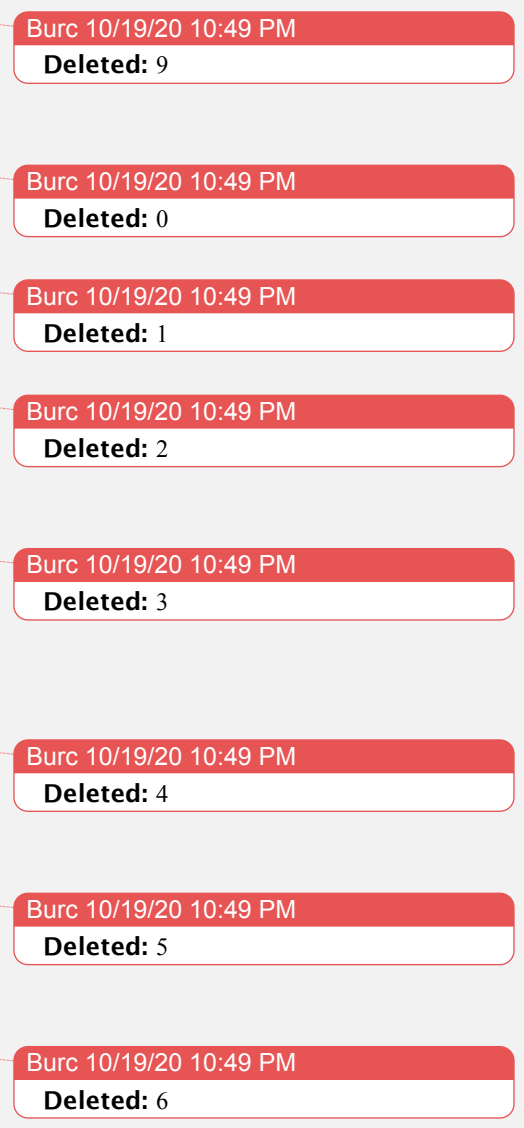


Figure 1 Legend. IgG Levels Overall, by Baseline Factors and Disease Severity. IgG levels were measured at a median [IQR] time of 66 [63.5-73] days from initial COVID19 diagnosis. See Supplementary Table 2 for detailed summary statistics.

667 Mod=Moderate, $\mathrm{Sev}=$ Severe .

Figure 2 Legend. COVID-19 as Life-changing Trauma (CALCT) Scores Overall, by

Baseline Factors and Disease Severity.

671 Psychological measures were assessed around 2 months from initial COVID-19

672 diagnosis. See Supplementary Table 4 for distribution of responses to the three CALCT

673 survey items. Mod=Moderate, $\mathrm{Sev}=$ Severe.

Table 1. Baseline Characteristics and Disease Severity by Endpoint

\begin{tabular}{|c|c|c|c|}
\hline & & $\begin{array}{l}\text { Serology } \\
(\mathrm{N}=47)\end{array}$ & $\begin{array}{c}\text { Psychological } \\
\text { Survey } \\
(\mathrm{N}=41)^{1}\end{array}$ \\
\hline \multirow[t]{2}{*}{ Sex } & Women & $28(60 \%)$ & $23(56 \%)$ \\
\hline & Men & $19(40 \%)$ & $18(44 \%)$ \\
\hline \multirow[t]{3}{*}{ Age } & $0-29$ & $9(19 \%)$ & $7(17 \%)$ \\
\hline & $30-59$ & $25(53 \%)$ & $21(51 \%)$ \\
\hline & $60+$ & $13(28 \%)$ & $13(32 \%)$ \\
\hline \multirow[t]{4}{*}{ Education Completed } & Elementary School & $11(23 \%)$ & $10(24 \%)$ \\
\hline & Middle/High School & $15(32 \%)$ & $13(32 \%)$ \\
\hline & University or Higher & $18(38 \%)$ & $18(44 \%)$ \\
\hline & Not Reported & $3(6 \%)$ & \\
\hline \multirow{2}{*}{$\begin{array}{c}\text { Any Symptom } \\
\text { Reported at the Time } \\
\text { of Diagnosis }\end{array}$} & No & $10(21 \%)$ & $8(20 \%)$ \\
\hline & Yes & $37(79 \%)$ & $33(80 \%)$ \\
\hline \multirow{2}{*}{$\begin{array}{c}\text { Fever/History of } \\
\text { Fever Reported at the } \\
\text { Time of Diagnosis }\end{array}$} & No & $25(53 \%)$ & $23(56 \%)$ \\
\hline & Yes & $22(47 \%)$ & $18(44 \%)$ \\
\hline \multirow[t]{2}{*}{ Comorbidity $^{2}$} & No & $32(68 \%)$ & $28(68 \%)$ \\
\hline & Yes & $15(32 \%)$ & $13(32 \%)$ \\
\hline \multirow[t]{2}{*}{ Disease Severity $^{3}$} & Mild/Moderate & $38(81 \%)$ & $33(80 \%)$ \\
\hline & Severe/Critical & $9(19 \%)$ & $8(20 \%)$ \\
\hline
\end{tabular}

676

677

678

679

680
${ }^{1}$ Of the 47 individuals who provided blood samples for serology testing, four cases were not invited to respond to the survey (one $<18$ years old, and three not fluent in local language) and two declined to participate in the survey.

${ }^{2}$ Most frequently reported chronic diseases were hypertension $(\mathrm{N}=9)$ and diabetes $(\mathrm{N}=5$, two with concurrent hypertension).
Burc 8/27/20 1:32 PM

Formatted Table

Comment [27]: To address question by Reviewer \#1, this included

chronic/recurring comorbidities, and hence, included seasonal allergies, but not acute infectious conditions such as common cold/flu. However, based on patient records, no specific acute infectious conditions/disease were noted. 
$681{ }^{3}$ Disease severity was defined as critical (requiring intensive care), severe (requiring 682 oxygen therapy, but otherwise stable) and mild/moderate (all other cases including 683 asymptomatic cases).

684 
Table 2. Descriptive Statistics and Correlations Between the Measured Psychological

\begin{tabular}{|c|c|c|c|c|c|c|c|c|c|c|}
\hline Process & $\mathrm{M}$ & SD & 1 & 2 & 3 & 4 & 5 & 6 & 7 & 8 \\
\hline $\begin{array}{l}\text { 1. COVID-19 as Life-changing } \\
\text { Trauma (CALCT) }\end{array}$ & 3.17 & 1.41 & & & & & & & & \\
\hline 2. Negative Emotions & 2.61 & 1.25 & $0.54^{* *}$ & & & & & & & \\
\hline 3. Perceived Discrimination & 2.48 & 1.30 & $0.54^{* *}$ & $0.24^{\mathrm{ns}}$ & & & & & & \\
\hline $\begin{array}{l}\text { 4. Global Health before } \\
\text { Diagnosis }\end{array}$ & 4.45 & 0.72 & $-0.02^{\mathrm{ns}}$ & $-0.07^{\mathrm{ns}}$ & $0.09^{\mathrm{ns}}$ & & & & & \\
\hline $\begin{array}{l}\text { 5. Global Health after } \\
\text { Diagnosis }\end{array}$ & 4.21 & 0.83 & $-0.15^{\mathrm{ns}}$ & $-0.20^{\mathrm{ns}}$ & $-0.17^{\text {ns }}$ & $0.42^{* *}$ & & & & \\
\hline 6. Pro-social Tendencies & 4.39 & 0.97 & $0.25^{\mathrm{ns}}$ & $0.28^{\mathrm{ns}}$ & $0.18^{\mathrm{ns}}$ & $-0.20^{\mathrm{ns}}$ & $-0.16^{\mathrm{ns}}$ & & & \\
\hline $\begin{array}{l}\text { 7. Perceived Importance of } \\
\text { Protective Measures }\end{array}$ & 4.42 & 1.00 & $0.24^{\mathrm{ns}}$ & $0.09^{\mathrm{ns}}$ & $0.09^{\mathrm{ns}}$ & $0.14^{\mathrm{ns}}$ & $-0.12^{\mathrm{ns}}$ & $0.41^{* *}$ & & \\
\hline $\begin{array}{l}\text { 8. Future Stigma Related } \\
\text { Anxiety }\end{array}$ & 1.99 & 1.06 & $0.54^{* *}$ & $0.05^{\mathrm{ns}}$ & $0.80^{* *}$ & $-0.07^{\text {ns }}$ & $-0.06^{\mathrm{ns}}$ & $0.13^{\mathrm{ns}}$ & $-0.02^{\mathrm{ns}}$ & \\
\hline
\end{tabular}

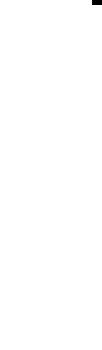

\footnotetext{
$\mathrm{M}=$ Mean, $\mathrm{SD}=$ Standard Deviation

Note. Standardized coefficients are shown. A psychological process score for a

participant reflects the corresponding average score of the survey responses (in the

ordinal scale of 1-5) used to measure that psychological process. Higher computed score

indicated stronger experience of COVID-19 as a life changing trauma, perceived higher

importance of preventive measures, stronger initial reaction to diagnosis, more positive

evaluation of general health, more perceived discrimination, higher post-traumatic

anxiety, or stronger anticipation of future COVID-19 related anxiety.

** $\mathrm{p}<0.001$; ns: non-significant $(\mathrm{p} \geq 0.05)$.
} 
Figure 1.TIF

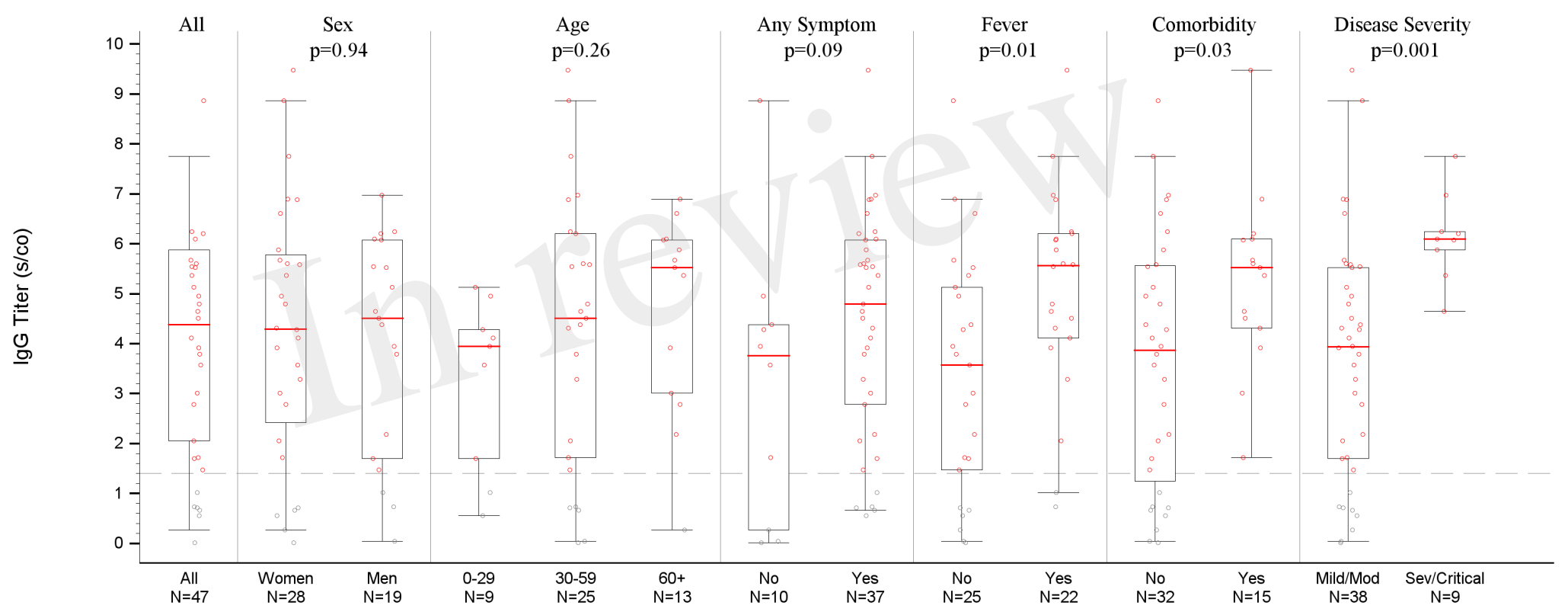


Figure 2.TIF

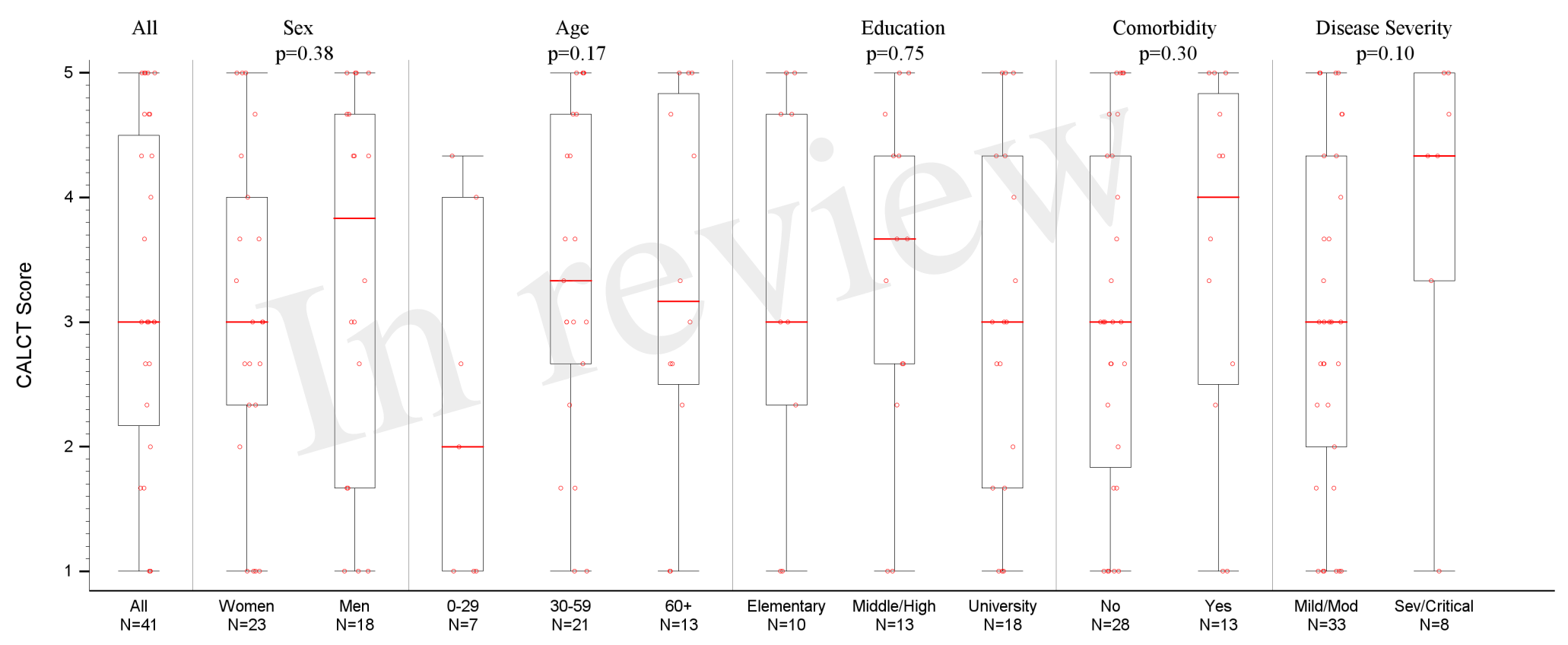

